\title{
Communications Equipment Operators
}

National Cancer Institute

\section{Source}

National Cancer Institute. Communications Equipment Operators. NCI Thesaurus. Code C122433.

Workers who operate telephone business systems equipment or switchboards, and/or provide information by accessing directories, assist customers with special billing requests. 